\title{
EL PERFIL COMPETITIVO LOCAL COMO FACTOR DETERMINANTE PARA EL DESARROLLO DE LA FLORICULTURA EN MADRID (CUNDINAMARCA)*
}

\author{
DANIEL HERNÁN SANTIAGO ROMERO** \& IVÁN MONTOYA RESTREPO*** \\ UNIVERSIDAD NACIONAL DE COLOMBIA
}

Recibido/ Received/ Recebido: 06-11-2011 - Aceptado/ Accepted / Aprovado: 11-12-2011

\begin{abstract}
Resumen
Este artículo describe los atributos del perfil competitivo del municipio de Madrid y establece las relaciones que, con dicho perfil, ha tenido el desarrollo de cultivos de flores. Se parte de la descripción de la situación actual de la industria de la floricultura en el país. Después, tras establecer los determinantes que desde la teoría se han propuesto para medir la competitividad de un territorio, se analiza el caso particular del municipio de Madrid y define los atributos competitivos que lo caracterizan. Se presentan las relaciones entre el perfil competitivo del municipio y la floricultura, mostrando cómo aquél, se convirtió en un factor determinante para dicha actividad económica. Finalmente se presentan las conclusiones del artículo, las cuales son antecedidas por la presentación de los elementos que representan la mayor fortaleza para el desarrollo económico del municipio en el futuro.
\end{abstract}

Palabras clave: Estrategia, Competitividad, Cluster, Agricultura.

\section{LOCAL COMPETITIVE PROFILE AS DETERMINANT FACTOR FOR FLORICULTURE DEVELOPMENT IN MADRID-CUNDINAMARCA}

\begin{abstract}
This article describes the competitive profile attributes of municipality of Madrid and establishes the relations that, with such profile, development of flower crops have had in its territory. The document begins describing the current situation of floriculture industry in the country. Then, after establishing the determinants proposed from theory to measure competitiveness in a territory, analyses the particular case of municipality of Madrid and defines the competitiveness attributes that characterize it. Finally, the relations among competitive profile and floriculture are presented, showing how it became a determinant factor for the development of this economic activity. The article conclusions are preceded by the presentation of the elements that represent major strengthen for economic development of the municipality in the future.
\end{abstract}

Keywords: Strategy, Competitiveness, Cluster, Agriculture.

\footnotetext{
* Trabajo de investigación realizado en el marco del desarrollo del trabajo final de la Maestría en Administración de la Universidad Nacional de Colombia, desarrollado por el primer autor bajo la dirección del segundo.

** Contador Público y Magíster en Administración (C), Universidad Nacional de Colombia. Correo electrónico: dhsantiagor@unal.edu.co.

**** Doctor en Ciencias Económicas, Profesor Asociado Universidad Nacional de Colombia. Correo electrónico: iamontoyar@unal.edu.co.
} 


\title{
O PERFIL COMPETITIVO LOCAL COMO FATOR DETERMINANTE PARA O DESENVOLVIMENTO DA FLORICULTURA EM MADRID-CUNDINAMARCA
}

\begin{abstract}
Resumo
Este artigo descreve as características do perfil competitivo no município de Madri, Colômbia, e estabelece as relações de referido perfil com o desenvolvimento de cultivos de flores em seu território. O documento parte da descrição da situação atual da indústria da floricultura no país, estabelece os pontos determinantes propostos para medir a competitividade de um território, analisa o caso particular do município de Madri e define as características competitivas que o caracterizam. Finalmente, apresenta as relações entre o perfil competitivo do município e a floricultura, mostrando como aquele se converteu num fator determinante para o desenvolvimento desta atividade econômica. As conclusões do artigo são antecedidas pela apresentação dos elementos que representam a maior fortaleza para o desenvolvimento econômico do município no future
\end{abstract}

Palavras chave: Estratégia, Competitividade, Cluster, Agricultura.

Santiago, D. \& Montoya, I. (2011) El perfil competitivo local como factor determinante para el desarrollo de la floricultura en Madrid Cundinamarca. En: Revista de la Facultad de Ciencias Económicas de la Universidad Militar Nueva Granada. rev.fac.cienc.econ, XIX (2)

JEL: L19, L79, N56.

\section{Introducción}

La toma de decisiones en las compañías de cualquier sector está siempre encaminada a alcanzar el mejor resultado posible. Uno de los enfoques predominantes en la teoría de la estrategia señala a la ubicación en una posición que permite el logro de un desempeño superior. Como parte de la explicación sobre este desempeño superior se afirma que la ubicación geográfica de las instalaciones de una compañía es un elemento determinante de la posición comercial que adquiere la organización dentro de su sector, de las ventajas que tiene frente a su competencia y, en general, del alcance de los objetivos y metas establecidos en la planeación estratégica de la misma. Los resultados de la decisión de escoger entre una ubicación u otra se verán siempre reflejados en los resultados de la operación del negocio.

Desde la perspectiva de Porter (1990), el éxito de una firma va, necesariamente, de la mano con las circunstancias de su ambiente, del espacio geográfico local donde desempeña sus actividades. Según Porter (1991) el origen de las ventajas competitivas empieza por determinarse con las elecciones que haga una firma, en términos de su industria, su posicionamiento geográfico, la configuración de sus actividades en una proposición de valor y sus resultados de mercado.

En el marco de la toma de decisiones estratégicas encaminadas a alcanzar el éxito para una firma, es labor preponderante determinar la competitividad del país, región o municipio donde se ubicarán las instalaciones de la compañía. De acuerdo con Porter (1990), existen cuatro atributos que permiten caracterizar o determinar con cierta claridad el perfil competitivo de una región. Estas cuatro fuerzas se representan gráficamente a través de una figura de diamante cuyas cuatro aristas son: las condiciones de los factores, las condiciones de la demanda, las condiciones de los sectores conexos y de apoyo; y las condiciones de estrategia, estructura y rivalidad de la empresa.

El sector de la floricultura en Colombia ha sido uno de los más importantes sectores en el nivel de las exportaciones nacionales. En el año 2009 exportó 
US\$1.050 millones frente a US\$1.114 millones exportados en 2007, sufriendo una disminución de casi $6 \%$ en este periodo de dos años. En 2010 el monto de ingresos presento una recuperación llegando a un total US\$1.240 millones.

En el presente artículo se realiza un análisis para determinar el perfil competitivo del municipio de Madrid (Cundinamarca), con el fin de establecer los elementos que favorecieron el desarrollo de los cultivos de flores en este territorio y señalar aquellos atributos que pueden constituirse como la mayor fortaleza del municipio de cara hacia el futuro económico local.

\section{Floricultura en Colombia}

De acuerdo con Asocolflores (2009b) para 2008 el área cultivada en flores en el territorio nacional ascendía a 7.509 hectáreas. Asocolflores (2009a) estableció que este total para el año 2009 fue de 7.200 hectáreas y, según datos de Asocolflores (2010a), el área cultivada para el año 2010 fue de 6.800 hectáreas, observándose una fuerte caída en el periodo 2008-2010, pasando de 7.509 hectáreas en 2008 a 6.800 en 2010, es decir, una disminución de 709 hectáreas, correspondientes al 9,4\%.

Del total de cultivos de flores existentes en el país, en el año 2009 el 76\% se encontraba en la Sabana de Bogotá, el $19 \%$ en Antioquia y el restante $5 \%$ en la zona Centro/Oriente de Colombia (Asocolflores, 2009b, 1).

En cuanto a las características que típicamente se observan en el producto están: "longitud, tamaño de la cabeza, apertura, problemas fitosanitarios, problemas físicos (tallo torcido, maltrato), curvatura y grosor del tallo, con tres presentaciones básicas: Sólidos, Mixtos y Bouquets."(Herrera \& Giraldo, 2004, $110)$; cuyo ciclo productivo presenta 5 picos de producción al año correspondientes a las temporadas de: San Valentín, Pascua, Día de la madre, Acción de Gracias y Navidad.

Los ingresos de los floricultores han tenido un comportamiento variable. En el año 2010 esta cifra ascendió a los US\$ 1.240 millones. El detalle de los ingresos para los años 2004-2010 puede ser observado en el Ilustración 1 , donde se observa una caída en los años 20072009 y apenas una recuperación en el año 2010.

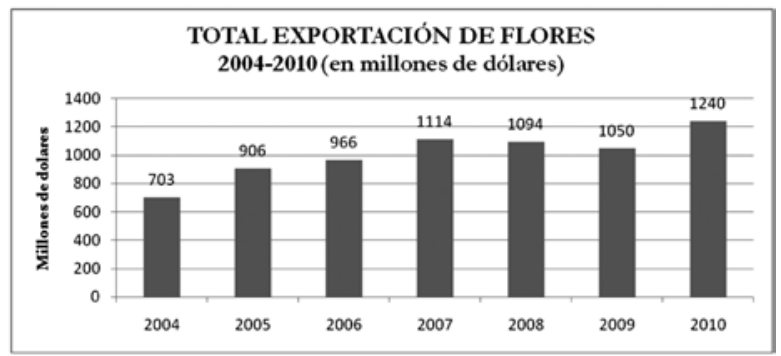

Ilustración 1. Total exportación de flores 2004-2010¹

Al observar al detalle el valor de las exportaciones según el mercado al que se dirigen, el comportamiento es muy estable como se observa en el Ilustración 2. La mayor parte de las flores vendidas se entregan a los Estados Unidos, donde el monto de los ingresos por ventas en este país fluctúan en forma similar al monto total de las ventas visto anteriormente. De esta manera, presentan una disminución de 75 millones de dólares entre 2007 y 2009 y una recuperación apenas en 2010.

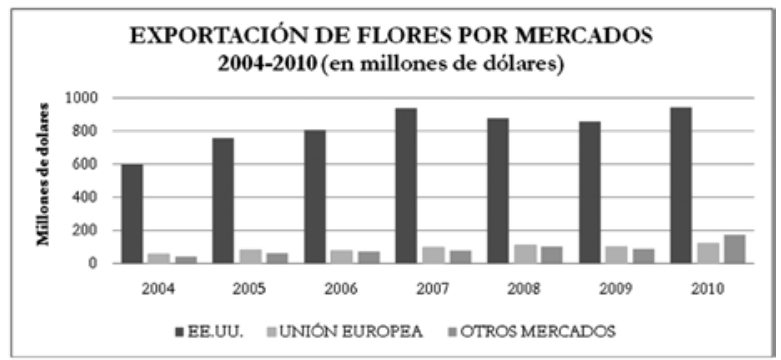

Ilustración 2. Exportación de flores por mercados 2004-2010²

En cuanto a los destinos de las flores en el extranjero, Estados Unidos pasó de recibir el 79\% del total de flores en 2009 al 76\% en 2010. Europa y los demás mercados tienen poca participación en el total de las exportaciones de flores. El detalle de los países destino de las exportaciones, según el porcentaje de participación en el total de la producción, se observa en la Tabla 1 que

Fuente: Elaboración de los autores con datos de Asocolflores (2009a, 2009b, 2010a).

2 Fuente: Elaboración de los autores con datos de Asocolflores (2009a, 2009b, 2010a). 
muestra cómo apenas el $24 \%$ del producto exportado no entra al mercado estadounidense y cómo, de los países que se reparten ese $24 \%$, cada uno cuenta con una muy pequeña participación, siendo el mercado ruso el segundo en importancia, apenas con un 5\%.

Tabla 1. Principales destinos de exportación de flores año $2010^{3}$

\begin{tabular}{|l|c|}
\hline \multicolumn{1}{|c|}{ PAÍS } & $\begin{array}{c}\text { PARTICIPACIÓN EN TOTAL } \\
\text { EXPORTADO }\end{array}$ \\
\hline ESTADOS UNIDOS & $76,0 \%$ \\
\hline RUSIA & $5,0 \%$ \\
\hline REINO UNIDO & $4,1 \%$ \\
\hline JAPÓN & $3,8 \%$ \\
\hline HOLANDA & $2,3 \%$ \\
\hline ESPAÑA & $2,2 \%$ \\
\hline CANADÁ & $2,1 \%$ \\
\hline OTROS & $4,5 \%$ \\
\hline TOTAL & $100 \%$ \\
\hline
\end{tabular}

En adición, “(...) el consumidor europeo gasta $€ 405$ al año en promedio por persona en la compra de flores, la recuperación de la economía rusa y la prohibición a la entrada de flores provenientes de Holanda a ese país por motivos sanitarios, permite que las flores colombianas penetren este mercado con éxito. Se presentan tendencias en los arreglos como las flores deshidratadas que se consiguen mediante la tecnología de la liofilización, pero los arreglos florales o bouquets siguen siendo los preferidos. La producción y venta de flores en Colombia, la marca, el gusto y preferencias de los clientes, quienes prefieren a Colombia como proveedor al conocer sistemas de gestión como el programa Flor Verde de Asocolflores por la conciencia social y ambiental que influye directamente sobre el sistema productivo de las empresas."(Ojeda, 2009, 14).

Debido a la importancia que tiene Estados Unidos en el mercado de las flores colombianas, la Ilustración 3 muestra el detalle sobre como la participación de los americanos en el mercado de las flores ha decrecido en los últimos años, pasando de $79 \%$ en 2008 al $76 \%$ en 2010 , es decir, una disminución de $3 \%$ en los últimos 2 años. Esto corresponde con las variaciones negativas que presentaron los ingresos provenientes de las ventas de flores a ese país y que fueron revisados en el análisis de la Ilustración 2.

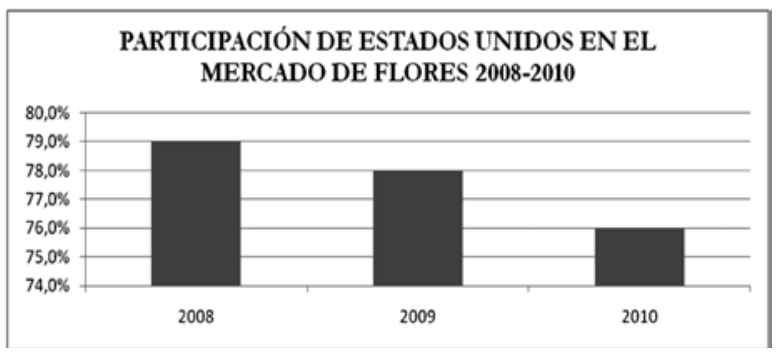

Ilustración 3. Participación de Estados Unidos en el mercado de flores años 2008-20104

Precisamente en relación con la importancia que tiene el comercio exterior para el sector de las flores, los tratados internacionales de libre comercio captan la atención de los empresarios, quienes, a través de Asocolflores, representan sus intereses en las negociaciones con los diferentes países, como es el caso del recientemente aprobado TLC con Estados Unidos y las preferencias arancelarias recibidas de este país a través del ATPDEA. Frente a otros tratados internacionales Asocolflores (2010b, 39) presenta los siguientes detalles:

Sobre el TLC con Canadá se afirma que éste será "el que genera un mayor impacto positivo a la floricultora colombiana, debido a que(...) Colombia tendrá muy pronto en vigencia este TLC y sus flores podrán ingresar a Canadá, a partir del primer día, sin pagar ningún tipo de arancel.”. Frente a la Unión Europea, "El proceso de negociación culminó exitosamente, lográndose el arancel cero a partir del primer día de vigencia del acuerdo para todos los tipos de flor.". Con los países EFTA se logró un mejor acceso de las flores colombianas a Suiza, Noruega, Islandia y Liechtenstein.

3 Fuente: Elaboración de los autores con datos de Asocolflores (2010a).

4 Fuente: Elaboración de los autores con datos de Asocolflores (2009a, 2009b, 2010a). 
En cuanto a los tipos de flores que se exportan, se encuentra que para el año 2010 la mayor parte de las flores exportadas son rosas (Ver Tabla 2).

Tabla 2. Principales tipos de flores exportadas año $2010^{5}$

\begin{tabular}{|l|c|}
\hline \multicolumn{1}{|c|}{ PRODUCTO } & $\begin{array}{c}\text { PARTICIPACIÓN EN TOTAL } \\
\text { EXPORTADO }\end{array}$ \\
\hline ROSAS & $33,0 \%$ \\
\hline CLAVELES ESTÁNDAR & $12,0 \%$ \\
\hline CRISANTEMOS Y POMPONES & $8,0 \%$ \\
\hline MINI CLAVELES & $6,0 \%$ \\
\hline ASTROMELIAS & $5,0 \%$ \\
\hline OTROS & $36,0 \%$ \\
\hline TOTAL & $100 \%$ \\
\hline
\end{tabular}

Para los floricultores tiene gran importancia el comportamiento de la moneda local frente al dólar debido a la alta relación comercial con el extranjero y a que casi la totalidad de la producción del sector está destinada a satisfacer mercados extranjeros. Desde el año 2007 los floricultores empezaron a enfrentar efectos negativos sus ingresos a causa de una tendencia de revaluación que todavía se mantiene. En su informe de actividades de 2010, la Asociación Colombiana de Exportadores de Flores, (Asocolflores), pone de manifiesto el fuerte impacto que sobre el gremio ha tenido la revaluación del peso, llamando la atención sobre la importancia que tiene en este sentido la participación de los representantes del gobierno en la toma de decisiones políticas y económicas que contrarresten o, por lo menos, disminuyan la agresividad con que la tasa de cambio ha caído (Asocolflores, 2010b, 42).

En el año 2011, el gremio floricultor ha continuado con sus permanentes llamados de atención a las autoridades nacionales para tomar medidas sobre la revaluación del peso, diciendo:

"Para el sector floricultor esta inundación de dólares es peor que la ola invernal que

\footnotetext{
5 Elaboración de los autores con datos de Asocolflores (2010a).
}

ha sufrido el país y que ha afectado a varios cultivos, mientras que la revaluación perjudica de manera grave a todos los floricultores ya sean pequeños, medianos y grandes (...). Pedimos que haya una política cambiaria clara para contar con una tasa de cambio consistente con el crecimiento económico. Así como hay reglas de juego claras para inversionistas extranjeros, pedimos reglas de juego claras para los empresarios que generan empleo formal y de calidad (...). La tendencia durante este año podría llevar al dólar a caer por debajo de los 1.700 pesos. Si esto sucediera haría inviable éste y otros sectores y nos veríamos obligados a un despido masivo de trabajadores del sector floricultor, lo cual repercutiría negativamente en la economía de 48 municipios del país donde hace presencia esta importante actividad agrícola primaria" (Asocolflores, 2011, 1).

Para comprender con mayor claridad los llamados de atención del sector, es importante observar el comportamiento de la Tasa Representativa del Mercado (TRM) en los últimos años, que se muestra en el Ilustración 4, que demuestra cómo, en lo que va corrido del siglo XXI, la TRM ha presentado una tendencia a la baja, disminuyendo considerablemente los ingresos recibidos en dólares como pago por el producto exportado. Con esta afección a sus resultados, los impactos negativos en las economías locales y regionales no se han hecho esperar, así como también en la vida de muchas personas que han perdido sus empleos con motivo de la insostenibilidad económica de algunas fincas cuyos dueños han optado por despedir a sus empleados y salirse del negocio.

El Estado colombiano no ha hecho oídos sordos a los llamados de los floricultores y les otorgó en el año 2008 créditos especiales para palear el efecto negativo de la revaluación. Sin embargo, los resultados de dichos créditos no fueron los esperados, tal como se describe a continuación: 


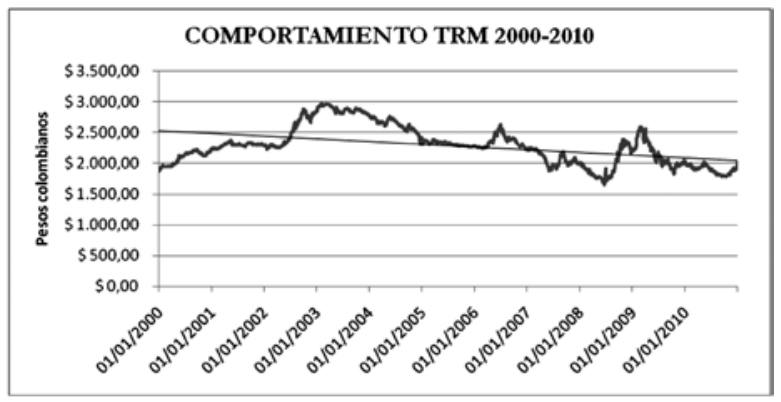

Ilustración 4. Comportamiento de la tasa representativa del mercado años 2000 a $2010^{6}$

"Con el propósito de aliviar los apremios de los exportadores, sometidos a las oscilaciones de la tasa de cambio en septiembre de 2008 el Fondo para el Financiamiento de Sector Agropecuario (Finagro) reglamentó una línea especial de crédito. A ella accedieron, vía Banco Agrario, un significativo número de productores agrícolas, especialmente floricultores. Dos años después, con \$224 mil millones desembolsados, el Estado está en serias dificultades para recuperarlos y el asunto ya llegó a los organismos de control... El plan de salvamento se puso de moda y un grupo de pequeños floricultores quiso hacer lo mismo. Pero así como a los empresarios más reconocidos del sector se les otorgaron préstamos con escasas garantías o dudosas informaciones financieras, a los de cultivos menores se les empezó a exigir garantías hipotecarias, incluso por un $40 \%$ de su valor comercial. De todos modos, el asunto se convirtió en un saco roto y se aprobaron créditos desde $\$ 14$ millones hasta $\$ 18$ mil millones, según el poder empresarial. Con el paso de los meses, el Banco Agrario se percató de que después de desembolsar tanto dinero necesitaba abonos a capital para garantizar su retorno. Pero sucedió lo que estaba cantado. Buena parte de los beneficiarios, sin modo de responder y con las mismas dificultades en sus exportaciones, empezaron a solicitar la refinanciación de sus prés- tamos. Finagro invocó su reglamento para evitar mayores concesiones y las empresas tuvieron que improvisar maniobras económicas para ponerse al día o definitivamente declararse en quiebra y buscar una salida al atolladero. Del mismo modo como la propuesta de la línea especial de crédito autorizada en 2008 se volvió famosa entre los floricultores y se accedió masivamente a ella con el argumento de preservar los empleos de sus trabajadores, también trascendió lo que terminó pasando con esos créditos" (Quevedo, 2010, 1).

\section{Competitividad}

Hablar de competitividad es remontarse más de tres siglos atrás con las teorías del comercio, y al mismo tiempo reconocer que todavía no existe un consenso sobre una única definición del concepto y que no existe una sola vía de medición de la misma (Benzaquen et ál, 2010). Kitson et ál (2004), citados por Lotero et ál $(2009,111)$, han tratado de acercarse al concepto de competitividad, manifestando que:

"Se asocia con la capacidad que posee una economía para producir bienes y servicios que cumplen con los test de los mercados internacionales, manteniendo simultáneamente altas tasas de crecimiento y unos elevados niveles de bienestar de la población. De acuerdo con tal definición, una región será más competitiva a medida que logre aumentar su capacidad de participación en el mercado (desplazando completa o parcialmente a otras regiones) y aumentando, en consecuencia, el ingreso de sus habitantes" (Lotero et al. 2009, 111).

Para Benzaquen et ál (2010) el concepto de competitividad ha evolucionado a través de la historia de acuerdo con la descripción que se presenta en la Ilustración 5. Además, manifiestan que en síntesis, la evolución de la teoría de la competitividad parte de dos teorías fundamentales: la teoría económica tra-

6 Fuente: Elaboración de los autores con datos de Banco de la República (2011). 
dicional y la teoría económica moderna. La primera está representada por el modelo de comercio internacional que se alinea con las teorías expuestas por Smith (1776), y la segunda por el modelo de la ventaja competitiva de las naciones, también conocido como el "diamante de la ventaja nacional" (estructurado por Porter (1990), del que se derivan los determinantes de la ventaja nacional competitiva, y de cuyo proceso de evolución se obtienen los dos estudios más reconocidos en el ámbito mundial que miden la competitividad de las naciones mediante la ejecución de sus modelos teóricos" el Informe de competitividad mundial y el Anuario de competitividad mundial, el primero desarrollado por el Foro Económico
Mundial (WEF, por sus siglas en inglés) y el segundo por el Instituto Internacional para el Desarrollo de la Capacidad de Gestión (IMD, por sus siglas en inglés)" (Benzaquen et al. 2010, 71).

Los desarrollos teóricos de Michael Eugene Porter en la estructuración de los conceptos y mediciones de competitividad han sido utilizados en diferentes instancias políticas, económicas y académicas. Para Porter el éxito de una firma va de la mano con las circunstancias de su ambiente. Según Porter (1991) el origen de las ventajas competitivas empieza por determinarse con las elecciones que haga una firma, en términos de su industria, su posicionamiento, la configuración de sus ac-

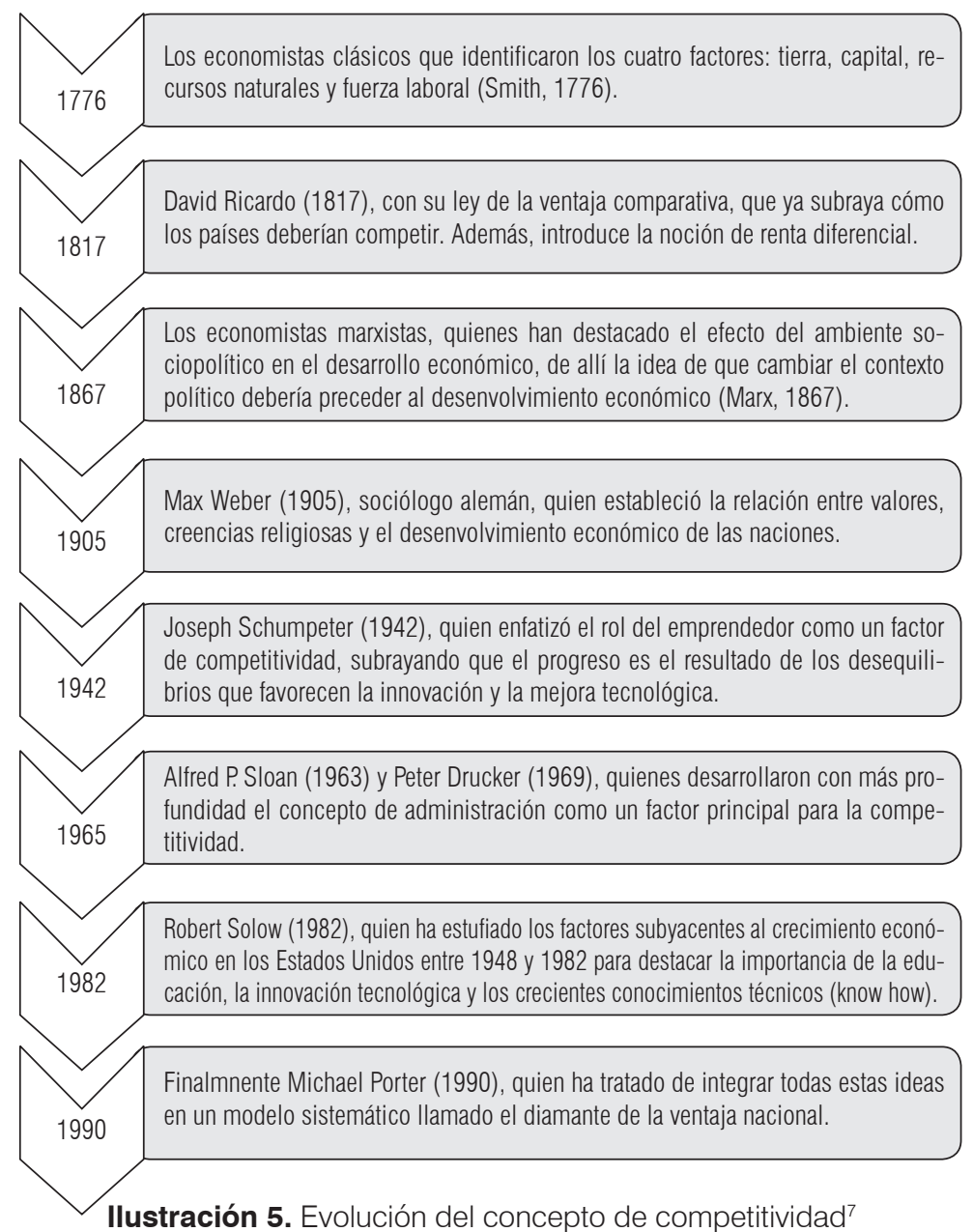

7 Fuente: Tomado y adaptado de Benzaquen et ál (2010, 71). 
tividades en una proposición de valor y sus resultados de mercado. Sin embargo, hay que tener presente que estas elecciones se enfrentan a restricciones inherentes a su posición inicial dentro de un mercado, la posición en que se encuentren sus competidores y la estructura del sector; así como la estructura interna de la firma.

El mismo Porter (1991) precisa cómo, desde el punto de vista de los recursos, el origen de la ventaja competitiva depende de ciertos recursos muy apreciables que la firma posea y que no son necesariamente recursos tangibles, son bienes intangibles como las capacidades, habilidades, reputación y similares. Sin embargo, la ventaja competitiva para una firma provendrá de algo más que sus recursos. Una aproximación a las razones de estas ventajas tendrá que incluir las condiciones internas de la firma, de la industria y del entorno en que esta opera. Además debe considerar los cambios en factores externos como las necesidades de los clientes, la tecnología, las actuaciones del gobierno y los insumos del mercado. Debe considerar que no solo será posible escoger de entre las estrategias de que se dispone actualmente, sino que también podrán crearse algunas nuevas, teniendo en cuenta los efectos de la aleatoriedad sobre la estrategia seleccionada.

Porter (1990) presentó la importancia que para la ventaja competitiva de las firmas tiene el entorno. La ventaja competitiva de una firma puede residir tanto en las características internas de la firma como en las características del entorno en que desempeña su actividad.

Las firmas crean y sostienen su ventaja competitiva gracias a su capacidad para mejorar continuamente, innovar y modernizarse a través del tiempo. Cuatro atributos del entorno de una firma tienen la mayor influencia sobre su habilidad para innovar y modernizarse. Estas cuatro fuerzas o atributos del entorno son: las condiciones de los factores, que corresponde a la posición de un territorio en cuanto a los factores de producción como la fuerza laboral y la infraestructura; las condiciones de la demanda, en particular la naturaleza de la demanda interna del mercado local para los productos o servicios prestados por la compañía; los sectores afines y auxiliares, es decir, la presencia o ausencia en el territorio de industrias proveedoras y otras industrias relacionadas que son, además, competitivas internacionalmente; y la estrategia, estructura y rivalidad de las empresas, que incluye las condiciones en el territorio que definen como las firmas se crean, se organizan y administran, así como la naturaleza de la competencia doméstica.

Estos cuatro factores han venido a conformar lo que Porter (1990) definió como el diamante de los determinantes de la ventaja competitiva de una nación. La representación gráfica del diamante de Porter puede observarse en la Ilustración 6.

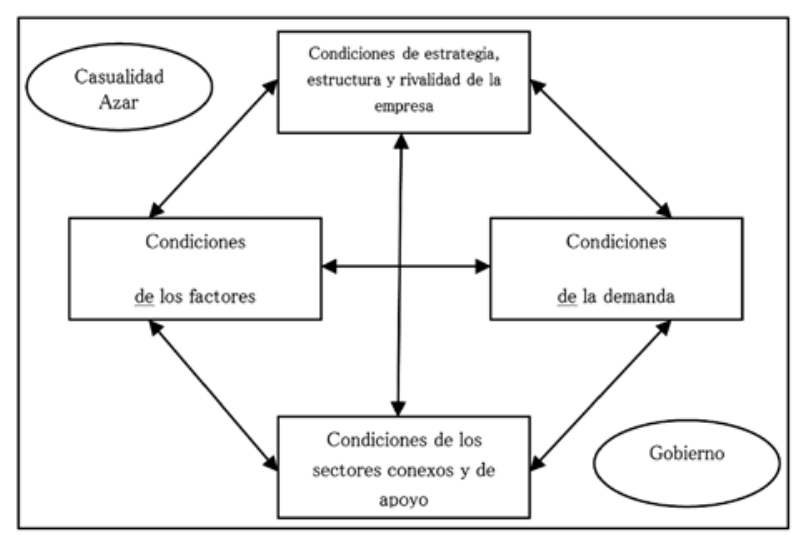

Ilustración 6. El diamante de Michael E. Porter ${ }^{8}$

Según Gómez et ál (2009), el diamante de Porter tiene aplicación a diferentes ámbitos como lo son una firma, un sector industrial, un país, una región o un municipio. De acuerdo con su interpretación:

"Michael Porter identifica cuatro áreas grandes denominadas "determinantes de la competitividad" y las representa en un modelo de diamante de cuatro aristas: Condiciones de los factores, dotación de factores especializados que son transferibles a sectores conexos y de apoyo; Condiciones de la demanda, en función de la composición de la demanda interna; Condiciones de los sectores conexos y de apoyo, en cuanto al acceso oportuno y eficaz a los principales insumos, unidades para coordinar o com-

8 Fuente: Adaptado de Porter (1990, 78). 
partir actividades en la cadena productiva y conformación de cluster de actividades, y Condiciones de estrategia, estructura y rivalidad de la empresa, referentes a cómo se crean, organizan y gestionan las compañías y la naturaleza de la rivalidad doméstica" (Gómez et al. 2009, 5).

Herrera \& Giraldo $(2004,111)$ explican que "el modelo del diamante busca dar respuesta al por qué de la productividad de empresas radicadas en ciertas zonas, analizando cuatro dimensiones que conforman el diamante de la ventaja competitiva."

Asimismo, Snowdon \& Stonehouse (2006) han dicho que el diamante de Porter trata de mirar el entorno inmediato donde una firma desempeña sus negocios, el cual influye sobre el mercado y las condiciones de competencia. Los factores que ilustra el diamante tienen una gran influencia sobre las condiciones que facilitan o entorpecen el desarrollo del potencial productivo de una empresa.

Por su parte, Krugman (1994) aclara que medir la competitividad en el ámbito nacional pierde sentido, ya que los países no compiten entre ellos, sino que corresponde más bien a un aspecto hacia el interior de la nación y no hacia el exterior, por lo cuál la importancia que adquieren lo regional y lo local en las mediciones de competitividad es preponderante.

Montoya (2010) afirma, citando a Porter (1991), que la teoría de la estrategia construida por este último incorpora el análisis de dos componentes: La atractividad de la industria o efecto industria y la posición relativa de la firma en la industria o efecto posicionamiento, que "implica la posibilidad de tener beneficios excepcionales en relación a sus pares en la industria. Estos beneficios derivan de una ventaja en la posición (se impone un precio más alto vía diferenciación, o se tiene un costo inferior mediante liderazgo en costos o enfoques). Porter sugiere que la estrategia se divide en actividades, a las cuales designa como unidad de análisis, lo que plantea que existen diferencias estructurales entre competidores que se ven reflejadas en los costos: escala, localización, integración vertical, regulación, antigüedad, etc, (a los que denomina Drivers)" (Montoya, 2010, 25).
Sin embargo, el mismo Montoya trata de establecer la principal crítica que se hace al modelo de Michael Porter al afirmar que:

"La crítica al enfoque de Porter radica fundamentalmente en que se concibe a la firma como un conjunto de actividades discretas. Esta suposición de consistencia interna, sugiere que los costos se establecen sobre las actividades y no de manera "holística" a la firma, y con una definición operacional de cadena de valor que establece un encadenamiento de las actividades. Así, para Porter la ventaja competitiva no se puede entender considerando la compañía como un todo, de forma que las auténticas ventajas en costos y diferenciación se deben buscar en la cadena de actividades que una empresa realiza para poder otorgar valor a sus clientes. Estas actividades identificadas por Porter son 5 actividades primarias (logística de entrada, operaciones, logística de salida, mercadeo y ventas y servicio), acompañadas de 4 actividades de apoyo (compras, desarrollo de tecnología, gestión de recursos humanos e infraestructura de la empresa)" (Montoya, 2010, 25-26).

Desde otra perspectiva sobre el rol que juega lo local en la competitividad de una firma, Requier-Desjardins et al. (2003) han dicho que la competitividad en los mercados mundiales descansa sobre específicas condiciones nacionales y locales que permiten la disponibilidad de mano de obra barata y recursos naturales, la cercanía con otros mercados y la ventaja competitiva proveniente de la agrupación de industrias específicas.

En contraste con lo planteado por Porter(1990), Requier-Desjardins et al. (2003) establecen cómo la globalización ha llevado a un nuevo lugar la internacionalización de las economías nacionales. En las dos décadas anteriores ha empezado a reconocerse que la globalización no puede darse sin tener en cuenta la variedad de los sistemas de producción nacional y local, estos no se pueden homogenizar y hacerse idénticos para todo el mundo. Ni siquiera los mercados se pueden asimilar, los hábitos de consumo en las 
naciones varían en algunas de sus variables. Por tanto, tratar plantear un modelo que recoja las particularidades de todas las naciones, regiones y territorios del mundo es tratar de homogenizar lo heterogéneo.

Para Requier-Desjardins et ál (2003) la importancia que tiene el factor localización es decisivo, así como cada sistema de producción es particular, así lo serán las ventajas de localizarse en cierta ubicación. La localización de las actividades económicas durante el desarrollo de la globalización ha llevado al agrupamiento o clustering de dichas actividades en locaciones específicas gracias a la facilidad que tiene el capital para moverse dadas las condiciones de globalización. Entre diferentes locaciones, la ventaja vendrá de aquellas donde estén disponibles los recursos y factores de menor costo.

El diamante de Porter (1990) representa las interacciones entre los factores de competitividad, las cuáles se dan en formas diferentes dependiendo de las características particulares de la nación donde se estén llevando a cabo las operaciones. Para Porter los procesos de reforzamiento entre los factores del diamante son mejorados por la proximidad geográfica, es decir, la existencia de clusters de industrias competitivas. Por tanto, en el nivel local, la competitividad reposa menos en la disponibilidad local de recursos naturales y los bajos costos y más en la calidad de los recursos humanos, las habilidades para la innovación y la calidad de los enlaces que existan hacia adelante y hacia atrás en la cadena de producción (Requier-Desjardins et al. 2003, 52).

En Porter (1998; 2000) la importancia de la localización es tal, que cuando un conjunto de firmas se agrupan en una región alcanzarán ciertas habilidades únicas, las cuales podrán preservar por un periodo largo de tiempo en los mercados globales. Dentro de estas habilidades se destacan las capacidades y know how desarrollados por las firmas en el contexto competitivo local, con lo cual todas las firmas que comparten un espacio geográfico común comparten también ciertos recursos y conocimientos que les proveerán ventajas competitivas como grupo de empresas (Tallman et al. 2004, 260).

\section{Perfil competitivo de Madrid-Cundinamarca}

El municipio de Madrid, como casi todas las poblaciones de la Sabana de Bogotá, tiene sus orígenes en las culturas indígenas que habitaban su territorio. Los chibchas habitaron el territorio actual del municipio, que entonces hacía parte del cacicazgo Tibaytata, dependiente del Zipa de Funza. Durante la conquista, entre 1559 y 1563, el encomendero Alonso Díaz cambio su nombre y fundó el nuevo Pueblo Indio de Serrezuela, nombre que se le dio al municipio por quedar junto a la única serranía de la Sábana. Finalmente, por disposición de la Asamblea de Cundinamarca se creó como distrito municipal en el año 1834. Su actual nombre honra la memoria de don Pedro Fernández Madrid, adoptado por la Asamblea de Cundinamarca mediante la Ley 14 de noviembre de 1875. El municipio abarca un área de 12.008 hectáreas y limita al noroccidente con Facatativa y El Rosal, al norte con Subachoque, al oriente con el cerro Manjui y los municipios de Tenjo y Funza, y al sur con Mosquera y Bojacá.

Conocer los detalles del municipio en este trabajo, no tiene sentido, si no se evalúan desde la perspectiva de la competitividad y desde la visión de los determinantes de la misma que Porter (1990) estableció. Por tanto, la definición del perfil competitivo de Madrid será como se muestra a continuación:

\subsection{Condiciones de los factores}

Geodésicamente, el municipio está ubicado a 04 grados, 44 minutos y 10.667 segundos de latitud norte, a 74 grados, 16 minutos y 47.060 segundos de longitud oeste y a una altitud de 2.550,090 metros sobre el nivel del mar, lo que representa una alta luminosidad durante todo el año al estar ubicado cerca al Ecuador.

"El municipio de Madrid está localizado sobre la cordillera oriental en el altiplano cundiboyacense, el casco urbano se encuentra a 2554 m.s.n.m. y distante a $29 \mathrm{~km}$. de Bogotá. Su tierra esta bañada por los ríos Subachoque y Bojacá, que se unen y for- 
man la laguna de La Herrera en el municipio de Mosquera. Por el municipio pasan transversalmente tres vías regionales; la carretera de occidente que atraviesa el casco urbano, la autopista Bogotá-Medellín que cruza entre las veredas de la Punta, Puente de Piedra y Chauta por el norte del municipio, y la actual variante. En medio de esta área que representa dos terceras partes del total del municipio, se localiza una chucua alrededor de la cual se desarrolla una gran parte de las actividades de floricultura. El municipio presenta una temperatura media anual de $13^{\circ}$, alcanzando una máxima media anual de $19.2^{\circ} \mathrm{C}$ y una mínima de $6.3^{\circ} \mathrm{C}$, condiciones que, junto con las condiciones de vientos y luminosidad a lo largo del año, originan un ambiente climático propicio para la producción agrícola. La precipitación es de $598 \mathrm{~mm}$. al año, siendo una de las zonas más secas del departamento" (Alcaldía municipal de Madrid, 2008, 16-23).

Las zonas planas del municipio están constituidas por suelos con alta aptitud para la agricultura y moderados requerimientos de nutrientes. La riqueza de los suelos y subsuelos presentes en Madrid, lo lleva a constituir una de las mejores tierras de Colombia, ofreciendo además condiciones de infraestructura envidiables en cuanto a su cercanía al mercado de consumo capitalino y a la infraestructura de comunicaciones nacional e internacional tanto terrestre como aérea, contando con el acceso a algunas de las mejores vías en el país en los niveles intra-regional, inter-regional e internacional (CIFA, 2000).

El impacto positivo que tiene la infraestructura disponible en el municipio para el desarrollo de la agricultura se puede corroborar en la utilización de predios para cultivar flores en el municipio. En el año 2008 cerca de 74 predios se dedicaban a esta actividad, esto de acuerdo con las cifras de predios registrados en la Alcaldía de Madrid. (Ver Tabla 3).
Tabla 3. Distribución de predios según actividad predial año $2008^{9}$

\begin{tabular}{|l|c|c|}
\hline \multicolumn{1}{|c|}{ DISTRIBUCIÓN DE PREDIOS SEGÚN ACTIVIDAD PREDIAL } \\
\hline ACTIVIDAD PREDIAL & $\begin{array}{c}\text { TOTAL } \\
\text { PREDIOS }\end{array}$ & PORCENTAJE \\
\hline RURAL CULTIVOS DE FLORES & 74 & $0,5 \%$ \\
\hline RURAL VIVIENDA & 1.733 & $12,8 \%$ \\
\hline RURAL INDUSTRIAL & 27 & $0,2 \%$ \\
\hline URBANO NO URBANIZADO & 143 & $1,1 \%$ \\
\hline URBANO VIVIENDA & 11.198 & $82,7 \%$ \\
\hline URBANO INDUSTRIAL & 49 & $0,4 \%$ \\
\hline AÚN SIN CLASIFICAR & 318 & $2,3 \%$ \\
\hline TOTAL & 13.542 & $100 \%$ \\
\hline
\end{tabular}

Por otro lado, en relación con los habitantes del municipio, que constituyen la mano de obra potencialmente disponible para trabajar en los cultivos de flores, la Alcaldía municipal de Madrid (2008) establece que el tamaño total de la población es de 67.042 habitantes para el año 2008, de los cuales, 58.024 viven en el área urbana del municipio $(86.42 \%$ ) y 9.018 en el área rural (13.58\%). Del total de habitantes $50.06 \%$ (33.566) hombres y $49.94 \%$ (33.476) mujeres.

Para revisar la oferta de mano de obra que posee el municipio se puede observar la distribución de población. Para el año 2008 (Ilustración 7) se observa cómo la mayor parte de la población que habita el territorio se encuentra dentro del rango de la población en edad de trabajar.

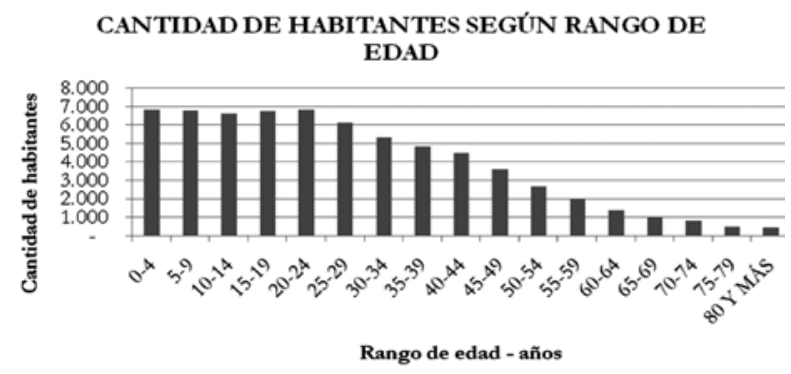

Ilustración 7. Cantidad de habitantes según rango de edad $(2008)^{10}$

\footnotetext{
9 Fuente: Tomado de Alcaldía municipal de Madrid (2008, 22).

10 Fuente: Elaboración de los autores con datos de Alcaldía municipal de Madrid (2008, 23-24).
} 
Sobre el nivel educativo alcanzado por la población de Madrid cabe señalar que en 2008 más del 70\% de la población se encontraba en un nivel bajo de calificación (máximo secundaria), que es el tipo de personal que en su mayoría requieren los cultivos de flores para la operación diaria (ver Tabla 4). Esto es coherente con el hecho de que en el municipio la mayoría del empleo es generado por las empresas exportadoras de flores, con 26.300 personas laborando en los cultivos y un total de 597 hectáreas cultivadas (Alcaldía municipal de Madrid, 2008).

Tabla 4. Nivel educativo de la población de Madrid (2008) ${ }^{11}$

\begin{tabular}{|l|c|}
\hline \multicolumn{1}{|c|}{ NIVEL EDUCATIVO } & PORCENTAJE \\
\hline PREESCOLAR & $5,2 \%$ \\
\hline PRIMARIA & $39,3 \%$ \\
\hline SECUNDARIA & $35,1 \%$ \\
\hline MEDIA TÉCNICA & $4,5 \%$ \\
\hline NORMALISTA & $0,1 \%$ \\
\hline TECNOLÓGICA & $4,3 \%$ \\
\hline PROFESIONAL & $4,4 \%$ \\
\hline POSGRADUAL & $0,2 \%$ \\
\hline NINGUNA & $6,8 \%$ \\
\hline TOTAL & $100 \%$ \\
\hline
\end{tabular}

Las condiciones de los factores que presenta Madrid son quizás condiciones envidiables por cualquier municipio del país, las condiciones de agua, suelo, infraestructura que posee el municipio, el acceso a la capital, las vías regionales y nacionales, la cercanía al aeropuerto más importante del país, la disponibilidad de mano de obra, aunque poco calificada, y demás factores, representan quizás, la mayor fortaleza que el perfil competitivo del municipio ofrece a los cultivos de flores.

\subsection{Condiciones de la demanda}

Las condiciones geográficas del municipio son las que le entregan la mayor fortaleza para que las flores puedan acceder fácil y rápidamente a la demanda externa. De acuerdo con la Alcaldía de Madrid, al referirse a los accesos regionales del municipio, "el casco urbano se localiza sobre la carretera de Occidente (que coincide con la calle 13 del Distrito Capital), que es una vía de orden nacional junto con la actual variante del municipio. Esta ha sido complementada con el eje paralelo conocido como la Autopista Bogotá-Medellín (que coincide con la calle 80 del Distrito Capital)"(Alcaldía municipal de Madrid, 2008, 20).

El análisis de las condiciones de ubicación geográfica del municipio, permite señalar que este factor es fundamental para otorgar un alto nivel de competitividad a Madrid. Esto en términos de las excelentes condiciones de acceso a los mercados internacionales, gracias al hecho de estar ubicado en la periferia de la capital del país y estar cerca del aeropuerto El dorado. Este está a menos de una hora de trayecto terrestre, ya sea por la autopista Bogotá-Medellín (que llega a la calle 80) o por la carretera de occidente (que llega a la calle 13), así las flores están disponibles para ser distribuidas a cualquier parte del mundo. Aspecto que además podría mejorar en el mediano o largo plazo, al encontrarse el municipio ubicado dentro de los planes de expansión poblacional del Distrito Capital dentro del proyecto Nueva Ciudad Occidente, por su localización dentro del eje industrial de occidente.

En cuanto a las condiciones de la demanda interna, Herrera \& Giraldo $(2004,111)$, explican que "la demanda interna no es sofisticada debido a la vocación exportadora del cluster. El consumidor local no está ni acostumbrado, ni dispuesto a pagar el precio internacional, ni da visiones estratégicas que anticipen tendencias globales. No se ha educado al comprador para que exija calidad, busque variedades exóticas o se fije en las condiciones de producción."

De esta manera, el factor de competitividad que corresponde a las condiciones de la demanda no estará relacionado exactamente con la satisfacción de demanda interna, sino, más bien, con las facilidades que ofrece el municipio para satisfacer la demanda

11 Adaptado de Alcaldía Municipal de Madrid (2008, 34). 
externa de flores. Serán entonces la facilidad para llegar al aeropuerto de Bogotá, la alta disponibilidad de vuelos internacionales y la eficiencia asociada al proceso de envío de las flores a los mercados americano y europeo, los elementos determinantes de la alta competitividad que, en cuanto a las condiciones de la demanda, ofrece el municipio de Madrid.

\subsection{Sectores afines y auxiliares}

Para hablar de los sectores afines y auxiliares de la floricultura en el municipio de Madrid, habría que retomar el esquema presentado por Herrera \& Giraldo (2004) que se muestra en la Ilustración 8, quienes lo explican así:

"El cluster (floricultor) incluye el sector de plásticos y de madera, proveedores de esquejes ${ }^{12}$ y semillas (compañías holandesas) para conseguir nuevas variedades de flor, $y$ a proveedores de pesticidas y abonos, los tres necesarios en el proceso productivo. En post-cosecha están los proveedores de químicos especiales para preservar la flor y de infraestructura fría para almacenarla; luego para el empaque intervienen proveedores de materiales como cartón, plástico para recubrir la flor, y cintas, cuya calidad afectan el trasporte. En distribución el trasporte aéreo es fundamental. Al final de la cadena están las comercializadoras encargadas de concentrar el producto, principalmente en Miami, y aunque no se encuentran en la misma zona del cluster, hay que mencionarlas porque son una extensión de las compañías floricultoras. Otros entes que participan en las actividades del cluster son los proveedores de servicios de $\mathrm{TI}$, las instituciones de colaboración (IC) y las instituciones públicas, todas con impacto considerable en el cluster" (Herrera \& Giraldo, 2004, 110).

Con respecto a la industria de los agroquímicos, que es una de las industrias que hacen parte del cluster, y que juegan un gran papel en la estructura de costos de producción, Ojeda (2009) describe el siguiente comportamiento de los empresarios floricultores,

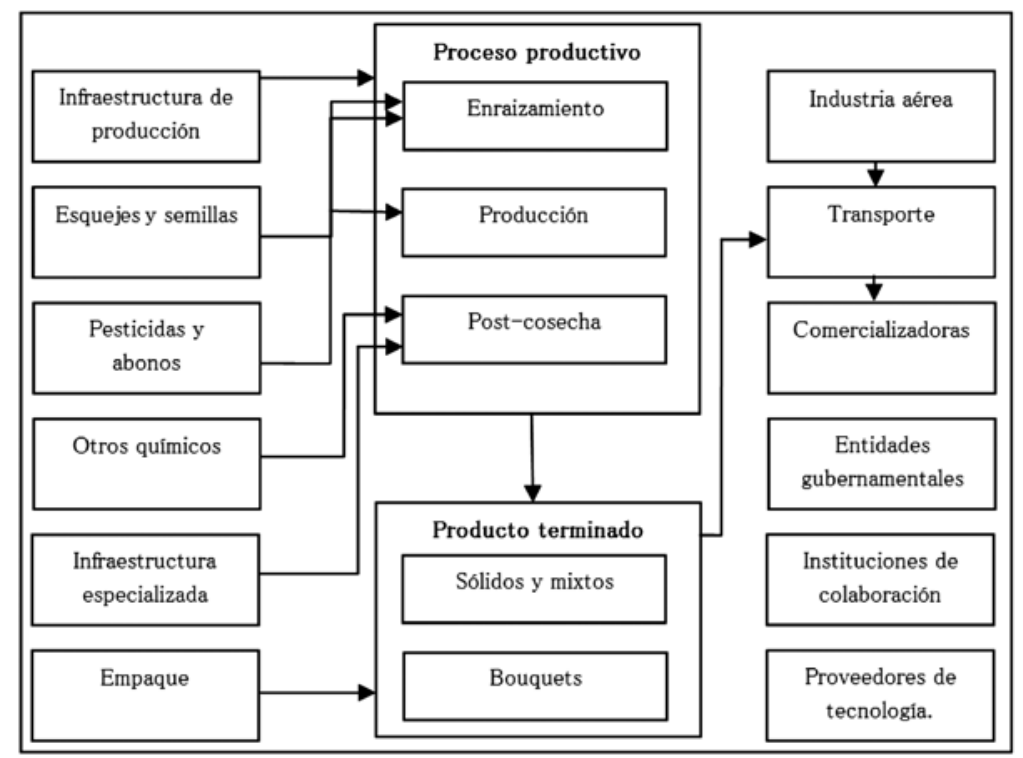

Ilustración 8. Anatomía del cluster floricultor colombiano ${ }^{13}$

Esqueje: Tallo o cogollo que se introduce en tierra para reproducir la planta.

Fuente: Adaptado de (Herrera \& Giraldo, 2004, 110). 
quienes tratan de recuperar financieramente las pérdidas provocadas por la revalorización a través de la disminución de costos, en este caso, del costo de los agroquímicos utilizados:

"Las empresas de flores intentan recuperar su rentabilidad y competitividad mediante prácticas como la reducción de costos en los insumos agroquímicos que representan en la estructura de costos alrededor del $6 \%$ del costo total de producción de las flores (...) Como parte de la iniciativa de reducción de costos, el sector floricultor ha aumentado el consumo de productos agroquímicos genéricos (...) Los floricultores han tomado como alternativa favorable el uso de estos productos, asumiendo en algunos casos el riesgo de sacrificar calidad por precio (trayendo) como consecuencia la disminución en la innovación de moléculas nuevas como resultado de la disminución de recursos para investigación y desarrollo, o de la falta de interés por parte de las empresas de agroquímicos de traer moléculas nuevas a mercados con poca diferenciación, en los cuales no se paga el costo de desarrollo por el uso de estos productos de marca" (Ojeda, 2009, 18-19).

El mismo Ojeda (2009) concluye, con respecto a la relación entre la competitividad del sector floricultor y la industria de los agroquímicos que:

"Se observa que en el mercado se encuentren productos genéricos con un valor en algunos casos de hasta el 50\% o menos del valor de los productos de marca, ocasionando a su vez una "guerra de precios" y por consiguiente erosión en el valor del mercado...La competitividad sectorial en la última década ha cobrado mayor relevancia para la agroindustria de flores, la tendencia a la baja en los últimos años de los precios internacionales de la flor y el cambio del dólar con respecto al peso colombiano, determinan el curso de acción, las decisiones y estrategias a tomar por parte de las empresas floricultoras, a fin de ser exitosos, competitivos en el mercado mundial y garantizar su permanencia en los mismos"(Ojeda, 2009, 20).

Entonces, la conclusión sobre este determinante será que "no hay una estrecha relación con los proveedores de madera y agroquímicos debido a la sensibilidad al precio; sólo con los proveedores de materiales de empaque, con alta especialización y competitividad, hay una relación cercana que ha generado beneficios mutuos. Con las industrias complementarias y afines, la más relevante es la industria de transporte aéreo, se encuentra una estrecha relación que se evidencia en la co-evolución de las dos desde los setentas" (Herrera \& Giraldo, 2004, 111).

\subsection{Estrategia, estructura y rivalidad de las empresas}

En cuanto a la estrategia, estructura y rivalidad de las empresas, se puede encontrar que en el municipio los cultivos de flores han sido creados en su mayoría por grupos familiares $y$, por tanto, gestionados y dirigidos como pequeñas empresas familiares. Así, la estrategia de los empresarios ha sido la de administrar sus compañías según un saber hacer familiar que han adquirido con la experiencia y que no están dispuestos a compartir con la competencia ni a poner a prueba confrontándolo con las innovaciones tecnológicas adquiridas o desarrolladas por las demás empresas del sector en el mundo.

"Hay empresas florícolas de tamaño pequeño, grande y mediano. Las empresas pequeñas tienen menos de 3 Has. en cultivo y son el $56 \%$ del total; las medianas tienen entre 3 y 10 Has. y abarcan el $29 \%$ del total; mientras que las empresas grandes, con más de 10 Has. en flores, son el $15 \%$ de las empresas florícolas y concentran la mayor parte de la producción y las exportaciones. Las tendencias en la concentración de la tierra tienen una estructura regionalmente diferenciada y ésta ha variado con el tiempo pasando de una concentración del 50\% entre las 10 empresas más grandes en 1981 a una concentración de menos del 40\%. Sin embargo, esta 
tendencia de aparente desconcentración responde más al mayor número de empresas que a que las empresas más grandes no hayan mantenido y aumentado sus áreas $y$, sobretodo, su participación en la producción y exportación. Esta tendencia a la concentración se da especialmente en algunos municipios de la Sabana de Bogotá como Madrid, Funza, El Rosal y Facatativa. Las empresas grandes tienen una superficie generalmente menor de 30 Has. y en el caso de la Sabana de Bogotá, un número de trabajadores entre 300 y 1.000 , determinado este por la etapa de desarrollo de la empresa y el grado de diversificación de la producción. La empresa mediana, con un área de entre 10 y 30 Has., tiene entre 100 y 300 trabajadores. Las pequeñas empresas tienen menos de 10 Has., menos de 100 trabajadores"(CENSAT, 2011, 6).

Sobre la organización del sector, se resalta la existencia de la Asociación Colombiana de Exportadores de Flores (Asocolflores), una organización gremial, sin ánimo de lucro, creada en 1973, con la misión de "representar y apoyar al sector productor-exportador de flores colombianas, para lograr su fortalecimiento y su desarrollo sostenible, armónico y competitivo, como fuente estable de empleo y generador de divisas, para el progreso del país y de sus afiliados" (Asocolflores, 2010b, 4).

Asocolflores a su vez impulsó en el año 2004 la creación del Centro de Innovación de la Floricultura Colombiana (Ceniflores) creado, según la información disponible en página web de la Asociación, "para promover, direccionar y articular recursos a fin de realizar investigaciones, desarrollo tecnológico, divulgación de resultados, estudios socioeconómicos, capacitación y servicios, que permitan fortalecer y mejorar la competitividad de la floricultura colombiana." (Asocolflores, 2011).

La caracterización de las empresas en el sector y en el municipio lleva a verlas principalmente como Pymes de origen familiar, administradas por sus dueños y por diferentes miembros de la familia, con una estrategia cerrada, en el sentido de querer siempre guardar su secreto corporativo adquirido a través del saber hacer y la experiencia de los años. Con esta rivalidad de puertas cerradas, la investigación y la innovación en el sector se limitan, pues no se comparten conocimientos entre las empresas ni tampoco con el gremio que las asocia (Asocolflores), de manera que cualquier tipo de investigación e innovación favorable para la productividad no es fácil de llevar a cabo debido a las restricciones impuestas por los mismos empresarios del sector. Entonces, en este marco cerrado, poco solidario y apático a la investigación, alcanzar estrategias de diferenciación que otorguen ventajas competitivas sostenibles se vuelve un reto (Herrera \& Giraldo, 2004).

\section{Relación floricultura - perfil competitivo de Madrid}

Después de describir la situación del sector floricultor, realizar la revisión teórica del concepto de competitividad y caracterizar el perfil competitivo de Madrid, es el momento de establecer las relaciones $y$ efectos que pudieron y pueden tener los atributos de municipio con el desarrollo de la industria de la floricultura en él.

Si bien, antes de empezar la realización de este estudio, la hipótesis inicial de los autores era que Madrid se había perfilado competitivamente como resultado de la instauración de las empresas floricultoras en el municipio, la realidad de la información obtenida demostró lo contrario, es decir, que las características de competitividad con que cuenta el municipio fueron tan favorables para la industria que motivaron a los empresarios a instaurarse en su territorio. En otras palabras, la floricultura se estableció en el municipio como consecuencia de las grandes ventajas competitivas que este representaba para el desarrollo del negocio, en cuanto a las condiciones de los factores de producción y las facilidades de acceso a la demanda internacional del producto.

Como se estableció anteriormente con la mayoría de los sectores auxiliares no se han creado estrechas 
relaciones comerciales, ante lo cual se puede decir que el municipio ha tenido en su haber éste tipo de negocios y que el problema ha radicado en la deficiente gestión desde la dirección de las diferentes fincas que no han consolidado fuertemente sus vínculos comerciales. De igual forma, la misma cercanía a la capital del país ha facilitado a los empresarios el contacto con el gremio de los exportadores (Asocolflores), permitiendo la negociación y comunicación con los más importantes representantes del gobierno y diferentes entidades del orden nacional y global. Los problemas relacionados con la gestión de las compañías como Pymes familiares que son, y las restricciones para compartir información que pueda mejorar la productividad del gremio a través de investigaciones sectoriales, son causados por las decisiones individuales de los empresarios y no por restricciones impuestas por el municipio de Madrid.

En definitiva, las características competitivas con que disponía y dispone el municipio de Madrid Cundinamarca favorecieron la entrada y permanencia de la floricultura en este, ya que los empresarios encontraron en él una serie de atributos que representaban ventajas comparativas y competitivas que, seguramente, otros municipios del país no podían ofrecer o, por lo menos, no en la misma proporción.

\section{Fortalezas del perfil competitivo para el futuro del municipio}

De los cuatro factores del perfil competitivo de Madrid es necesario revisar cuál es el factor o atributo, que se puede considerar como la mayor fortaleza del municipio frente a la floricultura y a otros sectores económicos que deseen, en un futuro, desarrollar sus procesos productivos en el territorio local.

De acuerdo con la información revisada, la dimensión del diamante que representa la mayor fortaleza del municipio es la que corresponde a las condiciones de los factores. Entre ellos: su localización geográfica, temperatura, disponibilidad de agua, calidad de suelos, alto porcentaje de población en edad de trabajar, mano de obra con diferentes niveles educativos, entre otros.
El análisis del perfil competitivo de Madrid permite destacar que, gracias a las condiciones de los factores que ofrece el municipio, las empresas de floricultura, $e$ incluso cualquier otro tipo de compañías, han encontrado y encontrarán facilidades de acceso a los mercados internacionales, a quienes toman decisiones político-administrativas en el país y a los avances tecnológicos generados desde la academia. De la misma manera, estas condiciones potencian la disponibilidad de servicios avanzados para la producción y de financiación a través de especializados servicios financieros. Finalmente, se obtiene un excelente clima social, político, económico y de seguridad que brinda la cercanía a la capital del país (Silva, 2005).

Este análisis es confirmado por Lotero et al. (2009) quienes, en su construcción de un índice global de competitividad desde la perspectiva de la geografía económica, llegaron a la conclusión de que la región Bogotá-Cundinamarca alcanza un mayor índice de competitividad comparado con las diferentes regiones del país debido a "la gran capacidad que tiene esta región para atraer recursos productivos a través de las inercias generadas por la aglomeración, de las ventajas que brinda su infraestructura y de las condiciones favorables en materia de geografía física" (Lotero et al. 2009, 129).

La CEPAL coincide con el planteamiento aquí expuesto afirmando que "la indivisible asociación entre Bogotá y el departamento de Cundinamarca se explica por la abierta metropolización de la primera, que desborda los límites políticos, administrativos, producto de la fuerzas centrífugas y centrípetas, características de las grandes urbes, o también por aquellos factores exógenos que afectan patrones productivos y comerciales, entre ellos, las políticas económicas y, recientemente uno de los de mayor peso, la globalización en todos los ámbitos" (CEPAL, 2007, 96). En el mismo sentido, se dirigen los resultados de CEPAL (2009) que coinciden con establecer la importancia que tiene competitivamente la ubicación geográfica para la competitividad de esta región y, por deducción, para Madrid.

La Comisión Económica para América Latina y el Caribe afirma que el liderazgo competitivo y productivo de la región conformada por Bogotá y Cundina- 
marca se ha mantenido en la última década. "Bogotá, como la principal área metropolitana, mantiene un extra liderazgo indiscutido, ratificado cuando se agrega con Cundinamarca. Este liderazgo esta fundamentado por las ventajas en fortaleza de la economía, y ciencia y tecnología, principalmente, y en capital humano e infraestructura" (CEPAL, 2009, 5).

Entonces, en este marco de alto potencial del municipio, debido a su ubicación en la zona geográfica de la región Bogotá-Cundinamarca, será necesario que las autoridades locales empiecen a desarrollar política pública orientada a fortalecer las capacidades competitivas señaladas e incluso a propiciar la aparición de algunas nuevas, de manera que se potencien los sistemas productivos locales, se facilite su adaptación a las necesidades del mercado y se atraigan nuevos sectores económicos a la región.

La atracción de nuevas actividades económicas al territorio local a partir del reforzamiento de las cualidades de competitividad con que cuenta el municipio será tarea prioritaria, teniendo en cuenta la situación en que se encuentra la industria floricultora con motivo de la disminución en sus ingresos y, en general, las dificultades que viene enfrentando, tal como se presentó en los apartes anteriores de este documento. "Sobre todo en el plano territorial es cada vez más importante diseñar instrumentos y políticas públicas de gestión dirigidas a estimular el aprovechamiento de los recursos locales endógenos para impulsar nuevos estilos de desarrollo basados en las potencialidades de las economías locales, como complemento indispensable de las políticas nacionales de desarrollo" (Silva, 2005, 83).

Finalmente, en palabras del mismo Silva (2005) con respecto a la importancia de la gestión pública de los territorios para propiciar la competitividad de las empresas, encontraremos las siguientes afirmaciones:

"Si bien es cierto que son las empresas las que compiten, su capacidad de competir solo se puede ver reforzada si el entorno territorial facilita la dinámica y si, por su parte, las empresas sienten la importancia de ser empresas "del territorio", a cuyos habitantes también se deben... La gestión pú- blica puede inducir el desarrollo de clusters, donde la colaboración y la asociatividad de las empresas son elementos centrales para impulsar su competitividad... (generando) ventajas comparativas avanzadas (principalmente en materia de conocimiento $e$ innovación), de particular relevancia para los grupos de Pymes concentrados en un territorio..., estas fuentes superiores de competitividad (el conocimiento y la innovación) surgen de la interacción entre empresas que son a la vez capaces de competir y de colaborar... Para construir territorios competitivos e innovadores es preciso aprovechar sus recursos endógenos, propiciando la asociatividad y la articulación públicosocial-privado con miras a flexibilizar los procesos productivos" (Silva, 2005, 86-88).

\section{Conclusiones}

La toma de decisiones en las empresas, sobre la ubicación estratégica de las instalaciones físicas de operación del negocio está altamente influenciada por las condiciones y atributos de la(s) zona(s) geográfica(s) que se está(n) considerando como alternativa(s) de decisión.

Las características de competitividad que puede brindar un territorio serán determinantes de las ventajas competitivas que una compañía pueda adquirir y mantener en el tiempo para abarcar la mayor parte del mercado y salir victoriosa frente a sus competidores.

Para los empresarios floricultores tomar en consideración los atributos con que cuenta el municipio de Madrid (Cundinamarca) para brindarles los mejores determinantes de competitividad en comparación con otras zonas del país constituye un elemento decisivo para el desarrollo de su negocio.

El perfil competitivo del municipio de Madrid, que incluye alta favorabilidad en las condiciones de los factores, acceso a la demanda internacional de flores, disponibilidad de sectores afines para todo el proceso productivo y de comercialización, y oportunidades de desarrollo de estrategias colectivas y gre- 
miales para fortalecer la flor colombiana en el mercado global; se ha convertido en un factor determinante, y quizás el más importante, para el desarrollo de la industria floricultora en el territorio municipal.

El municipio cuenta con diferentes características que lo hacen altamente competitivo y le otorgan un gran potencial dentro de la región Bogotá-Cundinamarca. Sin embargo, en un entorno de economía global, donde incluso los territorios locales se vuelven competidores por atraer a los inversionistas y a los empresarios, es necesario potencializar las envidiables condiciones de los factores con que cuenta Madrid y desarrollar, desde la administración local, acciones de política pública que lleven al fortalecimiento de estas condiciones de cara al futuro de la industria de las flores y a la atracción de diferentes sectores económicos con alto impacto social en los habitantes del municipio y en la economía local.

\section{Referencias}

Alcaldía municipal de Madrid. (2008). Plan de desarrollo municipal de Madrid Cundinamarca "Construyendo a Madrid con sentido social" 2008-2012. Obtenido el 26 de agosto de 2011 de: http://www.planeacion.cundinamarca.gov.co/BancoMedios/ Documentos\%20PDF/pdm 2008 2011\%20madrid.pdf

Asocolflores. (2009a). Colombian floriculture, 2009 stadistics. Obtenido el 31 de marzo de 2011 de: http://www. asocolflores.org/

Asocolflores. (2009b). Floricultura colombiana estadísticas 2009. Obtenido el 31 de marzo de 2011 de: http://mww.asocolflores.org/

Asocolflores. (2010a). Colombian floriculture 2010. Obtenido el 31 de marzo de 2011 de: http://www.asocolflores.org/

Asocolflores. (2010b). Informe de actividades 2010. Obtenido el 4 de abril de 2011 de: http://www.asocolflores.org/

Asocolflores. (2011). Boletín de presa No. 05 de 29 de abril de 2011. Obtenido el 1 de agosto de 2011 de: http://www. asocolflores.org/

Banco de la República. (2011). TRM serie histórica. Obtenido el 31 de octubre de 2011 de: http://www.banrep.gov.co/seriesestadisticas/see ts cam.htm\#tasa

Benzaquen, G., Del Carpio, L., Zegarra, L. \& Valdivia, C. (2010). Un índice regional de competitividad para un país. En: Revista CEPAL, 102: 69-86.

CENSAT. (2011). Las raíces de las flores, las deudas y los impactos de la floricultura en Colombia.. Obtenido el 27 de agosto de 2011 de: http://www.odg.cat/documents/enprofunditat/Deute_ecologic/Documento_5_Flores_en_colombia.pdf

CIFA. (2000). Documento técnico de soporte municipio Madrid -Cundinamarca Plan básico de ordenamiento territorial. Obtenido el 18 de agosto de 2011 de: http://www.car.gov. co/index.php?idcategoria $=3616$
CEPAL. (2007). Escalafón de la competitividad de los departamentos en Colombia 2007. En: Serie Estudios y perspectivas, 16: 1- 139.

CEPAL. (2009). Escalafón de la competitividad de los departamentos en Colombia 2006. En: Serie Estudios y perspectivas. 21: 1-107.

Drucker, P. (1969). The Age of Discontinuity. Nueva York: Harper \& Row.

Gobernación de Cundinamarca (2011). Mapa de infraestructura de transporte-Provincia de Sabana Occidente. Obtenido el 30 de agosto de 2011 de: http://www. planeacion.cundinamarca. gov.co/BancoMedios/Documentos\%20PDF/sabanaoccidente 08.pdf

Gómežz, M., Rosales, E. \& Gómez, A. (2009). El capital intelectual como factor de competitividad en la administración municipal. Caso región Toluca. Obtenido el 11 de agosto de 2011 de: http://octi.guanajuato.gob.mx/sinnco/formulario/MT/MT2009/ MT11/SESION3/MT113_MGOMEZ_185.pdfhttp://octi.guanajuato.gob.mx/sinnco/formulario/MT/MT2009/MT11/SESION3/ MT113 MGOMEZ 185.pdf .

Herrera, A. \& Giraldo, O. (2004). Uso de un modelo asociativo para el desarrollo del cluster floricultor colombiano. En: Revista de ingeniería, 19: 109-118.

Kitson, M., Martin, R. \& Tyler, P. (2004). Regional competitiveness: an elusive yet key concept?. En: Regional Studies, 38 (9): 991-999.

Krugman, P. (1994). Competitiveness: a dangerous obsession. En: Foreign Affairs, 73 (2): 28-44.

Lotero, J., Posada, H. \& Valderrama, D. (2009). La competitividad de los departamentos colombianos desde la perspectiva de la geografía económica. En: Lecturas de economía, 71: 107-140.

Marx, K. (1867). El capital: crítica de la economía política. México D.F.: Siglo XXI Editores.

Montoya, I. (2010). Una contribución a la comprensión de las estrategias deliberadas y emergentes de las organizaciones, desde una perspectiva evolutiva. Tesis de grado no publicada. Bogotá: Universidad Nacional de Colombia. Facultad de Ciencias Económicas.

Ojeda, H. (2009). Análisis de escenarios futuros de la industria de productos agroquímicos fitosanitarios de marca para la floricultura de la Sabana de Bogotá. Tesis de grado no publicada. Bogotá: Universidad Nacional de Colombia. Facultad de Agronomía.

Porter, M. (1990). The competitive advantage of nations. En: Harvard business review, march-april: 71-91.

Porter, M. (1991). Towards a dynamic theory of strategy. En: Strategic management journal, 12: 95-117.

Porter, M. (1998). Clusters and the new economics of competition. En: Harvard business review, 76(6): 77-90.

Porter, M. (2000). Location competition and economic development: local clusters in a global economy. En: Economic development quarterly, 14(1): 15-34.

Quevedo, N. (2010). Créditos raros en BanAgrario. En: El Espectador. Obtenido el 11 de diciembre de 2011 de: http://www. elespectador.com/economia/articulo-228771-creditos-rarosbanagrario

Requier-Desjardins, D., Boucher, F. \& Cerdan, C. (2003). Globalization, competitive advantages and the evolution of production systems: rural food processing and localized agri-food systems in Latin-American countries. En: Entrepreneurship and regional development: 15, 49-67. 
Ricardo, D. (1817). Principios de economía política y tributación. Madrid: Pirámide.

Schumpeter, J. (1962). Capitalismo, socialismo y democracia. México D.F.: Aguilar.

Silva, I. (2005). Desarrollo económico local y competitividad territorial. En: Revista de la CEPAL, 85: 81-100.

Sloan, A. (1963). My Years with General Motors.

Smith, A. (1776). An inquiry into the nature and causes of the wealth of nations. Nueva York: The Harvard Classics.

Solow, R. (1957). El cambio técnico y la función de producción agregada, Economía del cambio tecnológico. México, D.F.: Fondo de Cultura Económica.
Snowdon, B. \& Stonehouse, G. (2006). Competitiveness in a globalised world: Michael Porter on the microeconomic foundations of the competitiveness of nations, regions, and firms. En: Journal of international business studies, 37: 163-175.

Tallman, S., Jenkins, M., Nick, H. y Pinch, S. (2004). Knowledge, clusters and competitive advantage. En: The academy of management review, 29(2): 258-271.

Weber, M. (1905). The Protestant Ethic and the Spirit of Capitalism. Londres. 
